\title{
Levodopa in the treatment of Parkinson's disease: an old drug still going strong
}

This article was published in the following Dove Press journal:

Clinical Interventions in Aging

24 August 2010

Number of times this article has been viewed

\author{
Werner Poewe' \\ Angelo Antonini ${ }^{2}$ \\ Jan CM Zijlmans ${ }^{3}$ \\ Pierre R Burkhard ${ }^{4}$ \\ François Vingerhoets ${ }^{5}$ \\ 'Department of Neurology, Medical \\ University of Innsbruck, Austria; \\ ${ }^{2}$ Parkinson Department, IRCCS \\ San Camillo, Venice, and University \\ of Padua, Italy; ${ }^{3}$ Department of \\ Neurology, Amphia Hospital, Breda, \\ The Netherlands; ${ }^{4}$ Department of \\ Neurology, Faculty of Medicine and \\ University Hospitals of Geneva, \\ Switzerland; ${ }^{5}$ Neurodegenerative \\ Disorders Unit, Neurology \\ Department, CHUV, Lausanne, \\ Switzerland
}

\begin{abstract}
After more than 40 years of clinical use, levodopa (LD) remains the gold standard of symptomatic efficacy in the drug treatment of Parkinson's disease (PD). Compared with other available dopaminergic therapies, dopamine replacement with LD is associated with the greatest improvement in motor function. Long-term treatment with LD is, however, often complicated by the development of various types of motor response oscillations over the day, as well as drug-induced dyskinesias. Motor fluctuations can be improved by the addition of drugs such as entacapone or monoamine oxidase inhibitors, which extend the half-life of levodopa or dopamine, respectively. However, dyskinesia control still represents a major challenge. As a result, many neurologists have become cautious when prescribing therapy with LD. This review summarizes the available evidence regarding the use of LD to treat PD and will also address the issue of LD delivery as a critical factor for the drug's propensity to induce motor complications.
\end{abstract}

Keywords: levodopa, motor fluctuations, dyskinesia, Parkinson's disease

\section{Introduction}

After more than 40 years of clinical use, levodopa (LD) remains the gold standard regarding symptomatic efficacy in the drug treatment of Parkinson's disease (PD). ${ }^{1}$ Compared with other available dopaminergic therapies, dopamine replacement with LD is associated with the greatest improvement in motor function, as assessed by reduced scores in the Unified Parkinson's Disease Rating Scale [UPDRS])..$^{2-5}$ In addition, responsiveness to $\mathrm{LD}$ (required to exceed $25 \%-30 \%$ reduction in the motor part of the UPDRS) is a diagnostic criterion for PD. ${ }^{6}$ In clinical practice, LD slows the progression of disability as assessed by the Hoehn and Yahr staging system, ${ }^{7}$ and is associated with a reduction in mortality. ${ }^{89}$ Importantly, LD is one of the best tolerated drugs to treat $\mathrm{PD}$, particularly in the elderly population. ${ }^{10}$

However, long-term treatment with LD is often complicated by the development of various types of motor response oscillations over the day as well as drug-induced dyskinesia, a complication characterized by erratic involuntary movements. Such treatment-related motor complications eventually develop in the majority of patients and are found in about one-third of patients after only two years of exposure. ${ }^{11}$ Once established, motor complications are difficult to treat and can develop into a significant source of disability. In extreme cases, treatment-induced dyskinesias may completely annihilate the therapeutic benefit initially gained from the drug. Concerns about the potential induction of long-term motor complications have led many physicians to use LD in a restricted manner and reserve it as a second-line strategy. This approach has gained wide acceptance following clinical trials in early PD, showing significantly reduced risks
Correspondence:Angelo Antonini IRCCS San Camillo, Venice, and University of Padua, Italy Tel +39 04I 2207269

$\mathrm{Fax}+390412207469$

Email angelo3000@yahoo.com submit your manuscript | www.dovepress.com

Dovepress

DOI: $10.2147 /$ CIA.S6456 
of developing motor complications with dopamine agonists (DAs) as compared with LD monotherapy. ${ }^{2-5}$ While these trials have indeed established the potential of DAs to delay the onset of motor complications, they have also consistently demonstrated the superior symptomatic efficacy of LD, with a need for LD supplementation within the first 2-3 years in most patients started on a DA. ${ }^{2}$

Despite such evidence from clinical trials of the need for LD supplementation to maintain symptomatic control in early $\mathrm{PD}$, in general clinical practice, LD is often withheld beyond the time when symptomatic control with DAs has become insufficient. ${ }^{12}$ This results, in part, from patient perceptions. Information gleaned from physicians or the media may alert patients to the risk of dyskinesia associated with LD. The alarming nature of dyskinesia can, in turn, lead to a phenomenon labeled "dopa-phobia". ${ }^{12}$ These concerns have even been extrapolated to using single doses of LD in challenge tests, in order to prime the striatum putatively for subsequent dyskinetic responses to dopaminergic therapies. ${ }^{13}$

Increasing evidence now suggests that motor complications (particularly dyskinesia) associated with sustained LD therapy are a result of discontinuous and intermittent delivery of LD to the brain, resulting in nonphysiologic pulsatile stimulation of striatal dopamine receptors. Thus, the short half-life (90 minutes) of immediate-release LD formulations is thought to be the key factor in the pathogenesis of motor complications, rather than their induction being an intrinsic property of the LD molecule. ${ }^{1}$ This review will summarize the available evidence regarding the use of LD to treat PD and will also address the issue of LD delivery as a critical factor for the drug's propensity to induce motor complications.

\section{Efficacy of levodopa in early Parkinson's disease}

There have been a number of large-scale, long-term, LDcontrolled monotherapy trials of DAs in early Parkinson's disease on which strong conclusions about the relative effect size of LD compared with DAs can be based. The four-year randomized Comparison of the Agonist Pramipexole versus Levodopa on Motor Complications of Parkinson's Disease (CALM-PD) trial, compared initial treatment with pramipexole (0.5 mg three times daily) versus LD-carbidopa (100/25 mg three times daily), followed by open-label LD supplementation as required. ${ }^{2}$ The primary outcome measure was the time to first occurrence of dopaminergic complications, which included wearing-off (the re-emergence of PD symptoms due to the diminishing effect of LD), dyskinesias, "on-off" fluctuations (unpredictable fluctuations varying between symptoms being well controlled [on] to uncontrolled [off]), and freezing. Secondary outcome measures were changes in UPDRS scores and quality of life. Although initial treatment with pramipexole resulted in lower incidences of dyskinesia and wearing-off compared with initial treatment with LD, symptom control, as assessed by the UPDRS, was superior in patients treated with LD. From baseline to month 48, there was a worsening from baseline of $1.3 \pm 13.3$ (mean \pm standard deviation [SD]) points in UPDRS motor scores in the pramipexole group compared with an improvement of $3.4 \pm 12.3$ points in the LD group (treatment difference of 4.9 points, $P=0.001$ ) as shown in Figure 1A.

Interestingly, even though physicians in this trial had the option to use open-label LD supplementation to enhance symptomatic control, the group differences observed in the UPDRS motor and activities of daily living components between patients randomized to pramipexole or LD remained relatively uniform throughout the four years of the study. It is not definitely established why the UPDRS scores of the pramipexole group never caught up with the LD group, despite the option of open-label LD and other antiparkinsonian therapies, but it might be related to the lower dose of LD used in the supplemented pramipexole patients (434 $\pm 498 \mathrm{mg} /$ day) compared with those on LD monotherapy

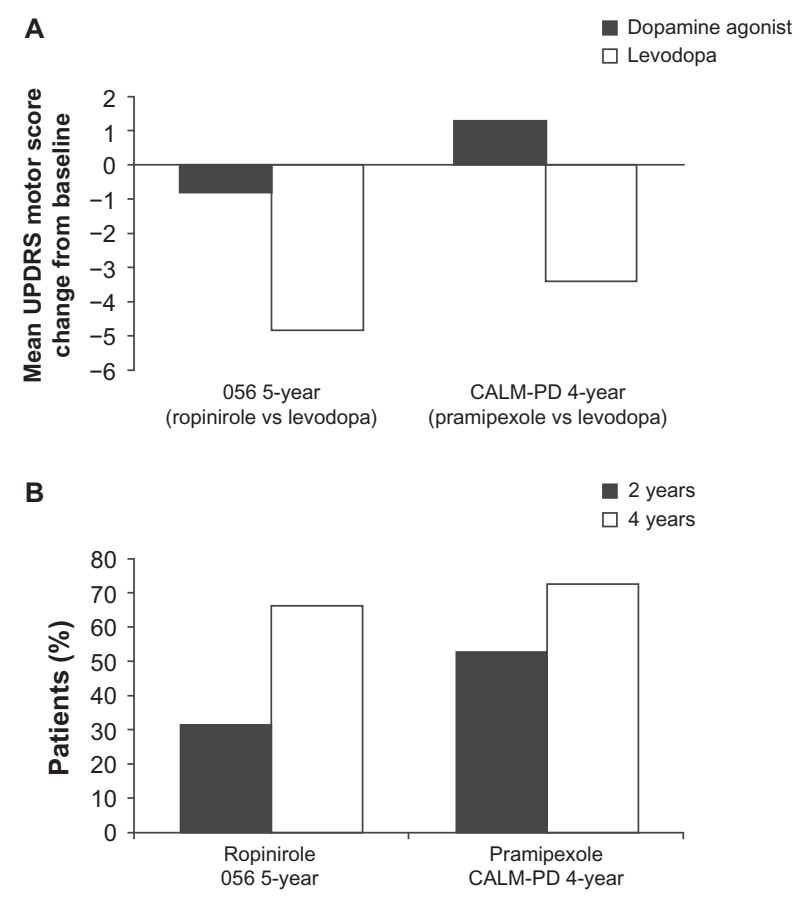

Figure I Mean \pm standard deviation change from baseline in A) Unified Parkinson's Disease Rating Scale (UPDRS) motor scores during the course of the five-year 056 and four-year comparison of the CALM-PD (Comparison of Agonist Pramipexole versus Levodopa on Motor Complications of Parkinson's Disease) trials by treatment assignment. B) Percentage of patients receiving monotherapy with dopamine agonists requiring levodopa supplementation after two and four years of treatment. ${ }^{2}$ 
(702 $\pm 461 \mathrm{mg} /$ day). Indeed, the calculated LD equivalent dose used in the supplemented pramipexole patients falls in the range of $468-584 \mathrm{mg} /$ day, which is notably less than the $702 \pm 461 \mathrm{mg}$ /day dose used in patients initiated on LD monotherapy. This indicates that combined treatment with a lower dose of LD and a DA is not equivalent to higher dose LD monotherapy in terms of symptomatic control, calling into question the concept of LD-equivalent doses of DAs.

In the five-year 056 study, which compared the safety and efficacy of ropinirole with that of LD, the primary efficacy measure was the occurrence of dyskinesia. ${ }^{3}$ Ropinirole was initiated at $0.25 \mathrm{mg}$ three times daily and increased weekly, as necessary, up to a maximum dose of $8 \mathrm{mg}$ three times daily. Levodopa was initiated at a dose of $50 \mathrm{mg}$ once daily and increased weekly, as necessary, to a maximum of $400 \mathrm{mg}$ three times daily. As in the CALM-PD study, if symptoms were not adequately controlled by the assigned study medication, patients could receive supplementary LD, administered in an open-label fashion. In this study, $84 \%$ of all patients initiated on ropinirole monotherapy either required LD supplementation (427 $\pm 221 \mathrm{mg} /$ day) or dropped out of the study. Although this study demonstrated a reduced incidence of dyskinesia for ropinirole versus LD ( $20 \%$ versus $45 \%$, respectively), this benefit was once again found to be at the expense of symptomatic control. In those patients who completed the study, there was a minimal improvement from baseline of $0.8 \pm 10.1$ points in UPDRS motor scores in the ropinirole group, contrasting with an improvement of $4.8 \pm 8.3$ points in the LD group (Figure 1A). Similar results confirming the superior efficacy of LD (in terms of UPDRS motor scores) were also observed in the two-year REAL-PET (ReQuip as Early Therapy versus L-dopa-Positron Emission Tomography) study of LD versus ropinirole monotherapy. ${ }^{14}$

The clinical relevance of such differences in UPDRS scores has been debated, but is vividly illustrated by the increasing rates of supplementation with open-label LD over the course of these double-blind comparative trials. At four years' follow-up in the CALM-PD trial, $72 \%$ of patients randomized to pramipexole monotherapy had required add-on LD to maintain symptomatic control, and this figure was $66 \%$ after five years in the 056 study with ropinirole (Figure 1B). ${ }^{2-5}$ The term "LD rescue", commonly used in this context, aptly describes the role of LD as the most efficacious drug at hand to control motor symptoms when other drugs begin to fail.

The recently published PELMOPET (Pergolide versus L-dopa Monotherapy and Positron Emission Tomography) study ${ }^{5}$ employed a strict pergolide $(0.75-5.0 \mathrm{mg} /$ day $)$ and LD
(150-1200 mg/day) monotherapy design in which no rescue therapy was allowed. At one year, there was a relatively small difference in favor of LD in the mean change from baseline in UPDRS motor scores $(-3.2$ points in the pergolide treatment group compared with -5.2 points in the LD treatment group). However, after three years of monotherapy, patients receiving pergolide had deteriorated below baseline by $2.8 \pm 9.8$ points, whereas patients receiving LD were still improved by $2.8 \pm 7.8$ points.

Taken together, these LD-controlled trials of DA monotherapy in early PD clearly show the need for LD supplementation to maintain symptomatic control.

\section{Delaying dyskinesias using initial monotherapy with dopamine agonists}

Current recommendations to initiate dopaminergic therapy in early PD with a DA, or even a monoamine oxidase inhibitor, are chiefly based on concerns about the evolution of motor complications characteristic of sustained LD therapy, most notably drug-induced dyskinesias. While the monotherapy trials cited above have clearly established a reduced dyskinesia risk with DA monotherapy, they have also shown that this is exclusively due to the delay in starting patients on LD. Indeed, PD patients initiated on a DA in these trials eventually required supplemental LD in order to maintain symptomatic control, and developed dyskinesias at an identical rate (albeit with a delay) to those started on $\mathrm{LD}$, leading to steady increases in dyskinesia rates from this point onwards. ${ }^{15}$ Therefore, the question remains as to whether the initial benefit of reduced motor complication rates can be maintained in the longer term when virtually all patients will be on combined drug treatment with DAs, LD, and possibly other agents. While none of the aforementioned comparative trials has been designed to assess outcomes under double-blind conditions for longer than five years, longer term, open-label, follow-up data are available. The Parkinson Study Group recently reported on the six-year outcomes of patient groups initially randomized to receive monotherapy with pramipexole or LD in the CALM-PD trial. Results of this study showed a persistent, statistically significant difference in overall dyskinesia rates of about $37 \%$ in those randomized to initial LD compared with $20 \%$ in those initiated on pramipexole $(P=0.004) .{ }^{16}$ Despite this, $90 \%$ of the population followed were on LD by six years. ${ }^{16}$ Similarly, 10-year outcomes reported by Lees et al ${ }^{17}$ comparing initial monotherapy with bromocriptine versus LD, along with Hauser et $\mathrm{al}^{18}$ from the 056 ropinirole study, also 
demonstrated a reduced overall incidence of dyskinesias with DAs in the long term. After 10 years, $45 \%$ of patients who were initiated with bromocriptine had dyskinesias compared with $54 \%$ of those started on LD (corresponding to an incidence rate for first dyskinesia occurrence of 145.3 versus 105.9 per 1000 patient-years after starting treatment with LD or DA, respectively). ${ }^{17}$ Similar figures were reported for the 10 -year follow-up of patients of the 056 study of ropinirole versus LD (52.4\% versus $77.8 \%, P=0.0457) .{ }^{19}$

While these results convincingly show long-term reductions in the incidence of dyskinesia with DAs compared with $\mathrm{LD}$, rates of dyskinesia do not necessarily reflect differences in dyskinesia-related disability between treatments. Hauser et $\mathrm{al}^{20}$ were the first to demonstrate differential functional significance of dyskinesias by asking patients to rate their involuntary movements as "troublesome" or "nontroublesome" in a modified "on/off" diary. When patients were subsequently asked to rate their recorded "on" or "off" times as functionally "good" or "bad" periods, $93.8 \%$ of "on" time with nontroublesome dyskinesia was considered functionally "good" time, whereas $84.9 \%$ of "off" time and $89.9 \%$ of "on" time with troublesome dyskinesia was considered "bad" time. ${ }^{20}$

In this respect, it is interesting to note that, at 10 and 14 years, respectively, neither the ropinirole nor bromocriptine studies found significant differences between treatment arms in the emergence of disabling dyskinesia. ${ }^{15,17,21}$ This finding was also observed in the CALM-PD study at four years, where the rate of disabling dyskinesias was low in patients treated with either pramipexole or LD. ${ }^{16}$ In addition, the recent STRIDE-PD (Stalevo Reduction In Dyskinesia Evaluation) trial comparing time to dyskinesia development in patients treated with either standard LD or a fixed combination of LD, carbidopa, and entacapone showed a less than $10 \%$ incidence of disabling dyskinesias in both groups. ${ }^{22}$

Furthermore, patient surveys have also indicated that patients with dyskinesias are less concerned about their dyskinesias than those who have not yet experienced dyskinesias, and that more than $80 \%$ of these patients prefer having dyskinesia over their PD symptoms. ${ }^{23}$ Since patients often tolerate mild dyskinesia well, the risk of dyskinesia should not cause physicians to delay LD initiation in patients whose PD symptoms cannot be sufficiently controlled with other treatments, regardless of patient age. Given these findings, the decision to initiate LD must be tailored to the patient's needs and should include proper counseling about the impact of dyskinesia and current options to minimize their incidence, including cautious LD dosing, or to treat them once they are present.

\section{Tolerability and safety issues}

In routine clinical practice, the benefits and risks of any anti-PD therapy must be weighed before prescribing the medication. LD therapy is generally well tolerated, and acute side effects include nausea, vomiting, and hypotension. ${ }^{24,25}$ As such, LD is generally started at a low dose to minimize these risks. With chronic use, the most common complications include wearing-off and dyskinesia, which can be troublesome for the patient (Figure 2). ${ }^{26}$ However, while the use of DAs is not associated with motor complications, a different array of side effects, including hallucinations, somnolence, and edema, are observed more commonly with these anti-PD medications (Figure 2). Dopamine agonists have also been linked to impulse-control disorders, such as pathologic gambling, hypersexuality, binge eating, or pathologic shopping, with about $13.7 \%-17.1 \%$ of patients on DA therapy showing signs of such disorders. ${ }^{27-29}$ While some of the adverse events associated with DA therapy are often perceived to be less bothersome for patients than LDinduced motor complications, others such as impulse-control disorders and sleep attacks, can have serious consequences for both the patient and their social relationships such that the advantages of delaying LD-associated risks by treatment with DAs may be negated. Therefore, the choice of therapy should be an individualized decision that takes into account the differential risk profiles of the various DA replacement strategies.

\section{Levodopa and progression of Parkinson's disease}

Another perceived risk of LD that still causes concern among many neurologists is related to its effect on disease

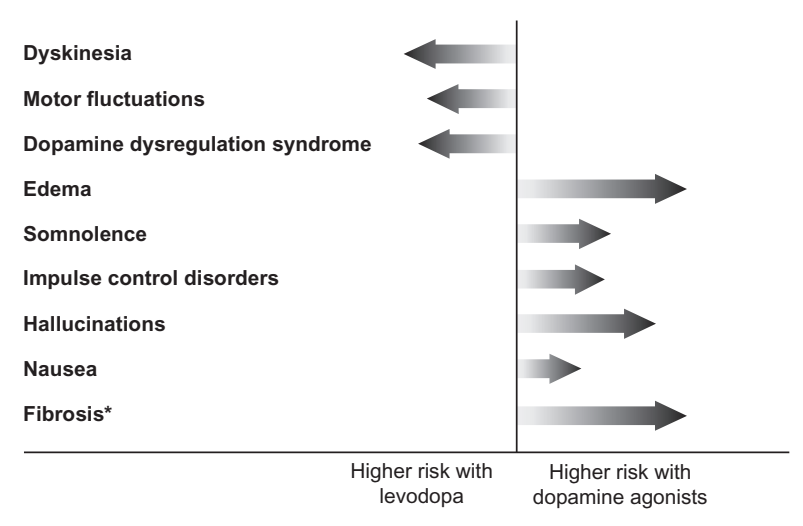

Figure 2 Comparison of the risk of motor complications and other adverse events associated with levodopa versus dopamine agonists. The length of the arrow reflects the extent of risk.

Note: *Ergot-derived dopamine agonists compared with levodopa. 
progression. In the early 1990 s, a number of in vitro studies demonstrated that high doses of LD can be toxic to dopaminergic neurons in cell culture, ${ }^{30-32}$ causing some PD specialists to recommend withholding LD for as long as possible..$^{33}$ Since that time, data have accumulated showing that LD may also have protective effects for cultured dopamine neurons, depending on experimental conditions, such as presence or absence of glia ${ }^{34,35}$ or ascorbic acid, ${ }^{36,37}$ as well as LD dose used..$^{38}$ In addition, many in vivo studies could not find evidence of LD-induced neurodegeneration in normal rodents,${ }^{39}$ primates, ${ }^{40}$ and nonparkinsonian humans,${ }^{41}$ and some have even reported neuroprotective effects of LD on midbrain dopaminergic neurons. ${ }^{42,43}$ Of particular interest are pathology reports from patients with essential tremor or dopa-responsive dystonia who had been chronically exposed to large amounts of LD over many years. None of these patients have shown evidence of substantia nigra degeneration at autopsy. $8,9,44$

After almost 40 years of established clinical use, the Parkinson Study Group recently conducted the first high-quality, randomized, placebo-controlled trial of $\mathrm{LD}$ to define better the effects of LD monotherapy on clinical progression in early PD. ${ }^{45}$ The ELLDOPA (Earlier versus Later Levodopa) study included 361 patients with early PD who were randomized to receive LD-carbidopa at a daily dose of 150/37.5 mg, 300/75 $\mathrm{mg}$, or $600 / 150 \mathrm{mg}$, respectively, or a matching placebo for a period of 40 weeks. The primary outcome was the difference in UPDRS scores between treatment groups at week 42 after withdrawal of treatment for two weeks. This endpoint was chosen to detect any potential underlying effect of active treatment on PD progression, with the assumption that a two-week washout period would remove all symptomatic LD effects, and any remaining differences in UPDRS scores between groups by this time would reflect treatment effects on disease progression. In addition, assessments of striatal dopamine transporter density using iodine-123-labeled 2 - $\beta$-carboxymethoxy-3- $\beta$-(4-iodophenyl)tropane ([$\left.{ }^{123} \mathrm{I}\right]$ $\beta$-CIT) single photon emission computed tomography were performed at baseline and at the end of study as a surrogate measure of progression of nigrostriatal terminal dysfunction. After a two-week washout period, the UPDRS motor scores in each group of LD-treated patients were still significantly improved compared with patients on placebo. This not only seems to exclude any evidence for negative effects of LD on the progression of PD but, on the contrary, suggests that treatment with LD results in a decline of UPDRS scores over time as compared with placebo. Nevertheless, there remains a possibility that a two-week washout could be insufficient for symptomatic effects of LD to wear off completely, thus precluding firm conclusions about the disease-modifying efficacy of LD from this study. Adding to this uncertainty, there was a significantly greater decline of striatal $\beta$-CIT binding in the high-dose arm of this trial compared with placebo in patients with abnormal scans at baseline. Interpretation of this finding is again confounded by the possibility of regulatory effects of $\mathrm{LD}$ on dopamine transporter binding or expression.

Integrating the currently available evidence from experimental studies and clinical trials, there is very little reason to assume that LD might hasten the clinical progression of $\mathrm{PD}$, and withholding the drug because of such fears from patients in clinical need of optimized symptomatic control is not warranted. Accordingly, US and European PD practice guidelines consistently recommend early use of LD in patients requiring initiation of dopaminergic treatment when the perceived dyskinesia risk is low, as is the case in the elderly. ${ }^{46}$

\section{Challenge of optimizing levodopa delivery}

The mechanism of action of LD is related to its activity as a prodrug for central dopamine and involves a number of critical steps, including gastrointestinal absorption, passage across the blood-brain barrier, neuronal uptake, and conversion to dopamine via enzymatic action of aromatic amino acid decarboxylase (AADC) (Figure 3), and eventually synaptic release of dopamine thus generated from exogenous LD. This sequence of events, needed for LD to exert its antiparkinsonian effect, is subject to a number of interfering processes, which can contribute to dose failures and longterm complications.$^{47}$ These include delayed gastric emptying and altered absorption of LD due to a competitive effect with ingested proteins at the level of amino acid transporters located in the gastrointestinal tract and the blood-brain barrier. Two major peripheral LD metabolic pathways, driven by the enzymes AADC and catechol- $O$-methyl transferase, significantly deplete the amount of $\mathrm{LD}$ reaching the brain. In addition, the short half-life (36-96 minutes) of LD is associated with fluctuating LD plasma levels, which eventually translate into fluctuating levels of synaptic dopamine derived from exogenous LD ${ }^{47}$ Consequently, for optimal benefit, LD has to be administered as multiple daily doses, but conventional three times daily regimens have not been found to be sufficient to establish constant plasma levels. ${ }^{48}$

In the earlier stages of the disease, oscillations in plasma levels are not clearly associated with fluctuations in 
(1) Swallowing oral therapy

Impaired swallowing (dysphagia) in advanced disease

(2) Stomach

Variable absorption of levodopa due to irregular gastric emptying

(3) Jejunum

Competition with dietary amino acids for active transport across the intestinal wall

(4) Peripheral tissues

Reduced levodopa bioavailability due to enzymatic breakdown by AADC and COMT

(5) Blood-brain barrier Competition for transport across the blood-brain barrier with large neutral amino acids limits the amount of levodopa reaching the striatum

(6) Striatum Conversion of levodopa to dopamine

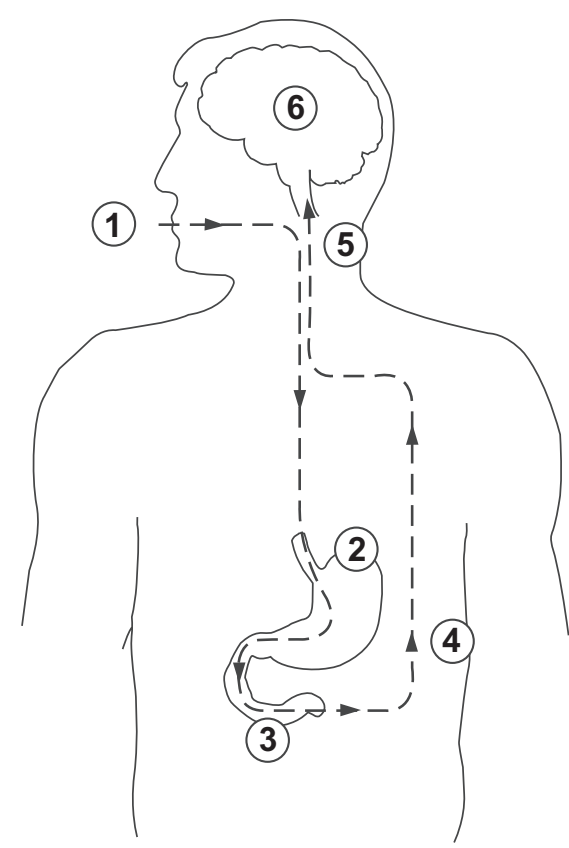

Figure 3 Oral levodopa therapy - hurdles on the route from the mouth to the brain. A number of factors impact on the progress of levodopa from the time of ingestion until it reaches the brain and is converted to dopamine.

Abbreviations: AADC, amino acid decarboxylase; COMT, catechol-O-methyl transferase.

motor function, presumably due to central "buffering" via intraneuronal storage in surviving nigrostriatal terminals, providing continuous stimulation even in the context of discontinuous exogenous delivery (Figure 4). However, with progressive loss of nigrostriatal terminals and accompanying changes in the central pharmacodynamics of LD, the clinical response to individual doses becomes progressively shortlived, resulting in wearing-off and "on-off"-type motor fluctuations. These motor fluctuations can be completely abolished by continuous intravenous infusions of LD, ${ }^{48}$ supporting the concept that providing a less pulsatile, more continuous, striatal dopamine receptor stimulation may be critical to restoring physiological motor processing in the striato-pallido-thalamo-cortical network in $\mathrm{PD}{ }^{49-53}$

The issue of continuous drug delivery is also relevant to the current understanding of mechanisms underlying the development of LD-induced dyskinesia. In animal models of $\mathrm{PD}$, administration of $\mathrm{D}_{1}$ or $\mathrm{D}_{2}$ agonists with short half-lives is associated with dyskinetic responses, ${ }^{54-57}$ while exposure to long-acting agonists does not induce dyskinesia. ${ }^{58-60}$ The same differences have also been observed in studies comparing pulsatile versus continuous delivery of the same dopaminergic agent. ${ }^{61,62}$ Such results are consistent with clinical studies of continuous infusions of DAs, such as apomorphine ${ }^{63,64}$ or lisuride ${ }^{65}$ which were found to downregulate pre-existing LD-induced dyskinesia. Indeed, when given as continuous intraduodenal infusions, marked reductions in dyskinesia have been reported for LD itself ${ }^{49,53,66,67}$ and the gel preparation of LD (Duodopa ${ }^{\circledR}$, Solvay Pharmaceuticals $\mathrm{GmbH}) .68,69$

Although pulsatile stimulation may not be sufficient to explain the mechanisms underlying the induction of dyskinesia completely, such observations highlight the need to optimize LD delivery in PD. The use of intravenous infusions is not feasible for chronic treatment, and intrajejunal infusion strategies are currently limited by high costs and the need for percutaneous gastrostomy. Other routes of LD delivery, eg, transdermal or transnasal, are currently under investigation but no nonenteral system has yet reached the market..$^{58-60,70,71}$ Previous attempts to improve oral delivery have included the development of sustained-release preparations of LD, but unfortunately randomized controlled studies have failed to reveal any difference between such formulations and standard LD with respect to long-term dyskinesia risk. ${ }^{72-74}$ Sustained-release LD preparations exhibit erratic absorption patterns and unpredictable plasma levels, ${ }^{67}$ resulting in dose failures as well as a delay in producing a clinical benefit. ${ }^{75}$ As such, the unpredictable absorption of these agents may not abolish high peak and low trough LD plasma levels that are associated with the development of dyskinesia.

Likewise, controlled trials have not clearly established superiority of sustained-release LD in terms of control of motor fluctuations. ${ }^{76}$ By contrast, prolonging the LD half-life via adjunctive treatment with a catechol- $O$-methyltransferase 

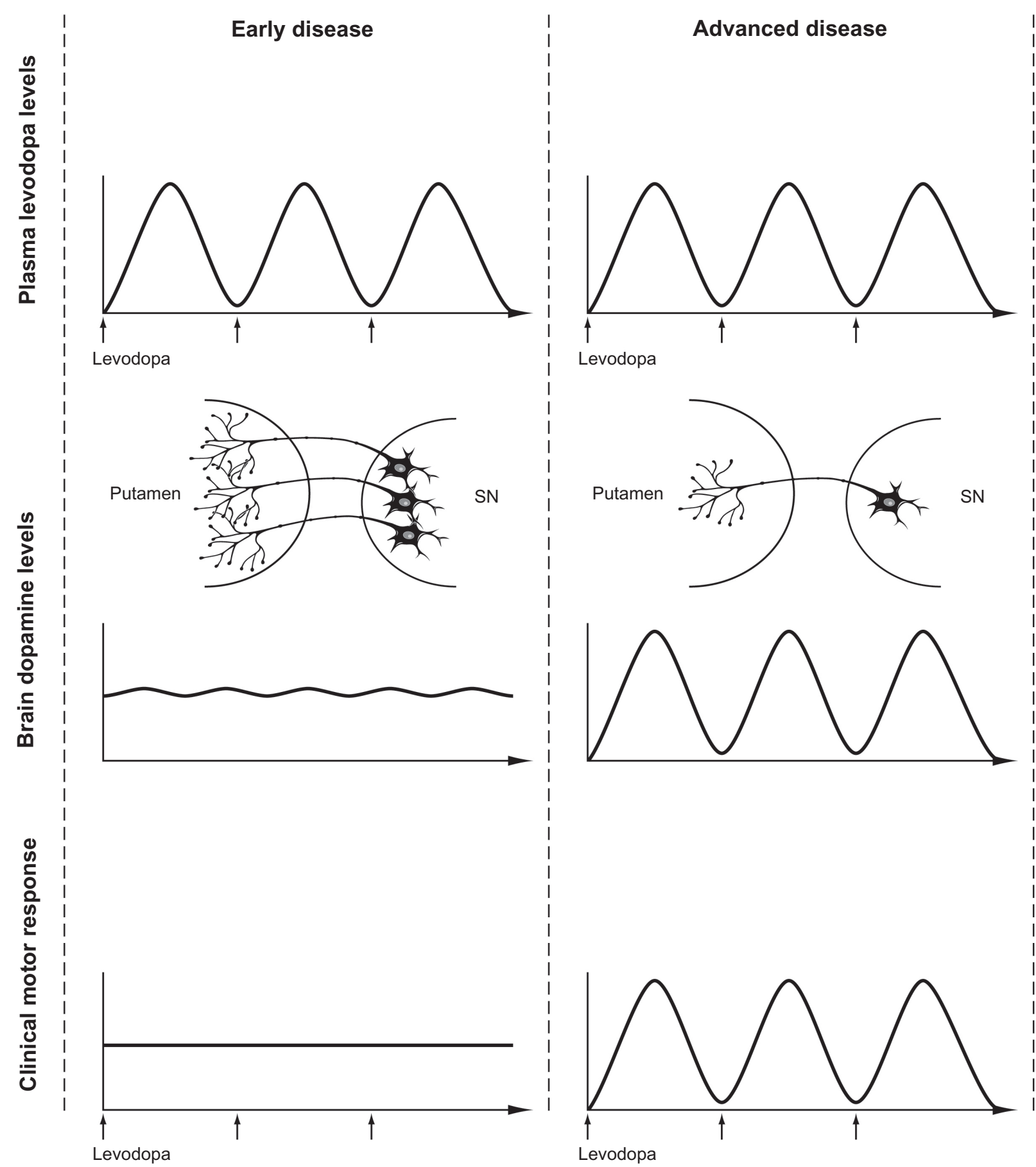

Figure 4 The evolution of levodopa-associated motor fluctuations. A comparison of plasma levodopa levels, brain dopamine levels, and the resulting motor response in early and advanced stages of Parkinson's disease is shown.

Abbreviation: SN, substantia nigra.

inhibitor, such as entacapone or tolcapone, has been found to be efficacious in reducing daily "off" time in a number of well-performed, randomized, controlled trials in PD patients with wearing-off. ${ }^{77-80}$

The established efficacy of entacapone in PD patients with wearing-off has raised the possibility that it may also be effective in reducing the risk of dyskinesias. However, results of the recent STRIDE-PD trial demonstrated that, in patients with early $\mathrm{PD}$ who are not experiencing wearing-off, a four times daily dose of a LD formulation comprising LD, carbidopa, and entacapone is not superior to conventional LD in delaying dyskinesias. ${ }^{22}$ The study investigators have 
proposed that entacapone may not have been administered frequently enough to achieve smooth levels of LD in the plasma. $^{22}$

As such, current research is focusing on the development of new LD formulations and delivery systems, such as novel controlled-release and transdermal preparations, which may improve delivery of LD in order to achieve smoother LD plasma levels. It remains to be seen whether these new LD formulations will prove effective in minimizing the risk of drug-induced motor complications.

\section{Conclusions}

Despite all recent advances in the medical management of PD, LD has remained the therapeutic gold standard in controlling the cardinal motor features of this illness. There is no evidence to support withholding LD for fear of hastening the progression of PD, although current three times daily regimens of standard oral LD carry a definite risk of inducing potentially disabling drug-induced involuntary movements. While dyskinetic responses are common with sustained LD therapy, the proportion of patients actually developing disabling and severe dyskinesia has been below $10 \%$ in a recent four-year randomized trial ${ }^{2}$ and below $20 \%$ in a 10 -year follow-up series. ${ }^{17}$ This should be taken into account when the physician discusses the potential use of LD with the patient, particularly in patients who require enhanced symptomatic control despite optimized treatment with DAs. Moreover, when making individual decisions on how to initiate dopaminergic treatment, the weighing of relative risks and benefits of starting with a DA or LD should not only consider dyskinesia risks but also risks for other side effects, including daytime somnolence, impulse-control disorders and, in the case of ergot-derived DAs, cardiac valvulopathy and other forms of potentially life-threatening fibrosis. ${ }^{27}$ In addition, individual needs for magnitude and speed of symptomatic improvement must be balanced against potential side effects. For over 40 years, LD has remained the cornerstone of PD therapy. Future endeavors will focus on optimizing delivery of LD in order to expand and improve the treatment options of PD.

\section{Acknowledgments}

The idea for the present review was originally discussed and formulated at a meeting of the Neuronet PD group, which was funded by an unrestricted educational grant from Novartis Pharma AG. We would like to thank Sevasti Bostantjopoulou, Luc Defebvre, Bulent Elibol, Joaquim Ferreira, Spiros Konitstotis, Jaime Kulisevsky, Rosario Luquin, Johannes
Van Hilten, and Marc Verin for their helpful comments. Editorial support for this manuscript was provided by Medicus International and funded by Novartis Pharma AG.

\section{Disclosure}

All authors are part of the Novartis-funded Neuronet PD group. WP has received consultancy and lecture fees from AstraZeneca, Teva, Novartis, GSK, Boehringer-Ingelheim, UCB, Orion Pharma and Merck Serono in relation to clinical drug development programs for PD. PRB has received a research grant from Novartis Pharma AG.

\section{References}

1. Olanow CW, Agid Y, Mizuno Y, et al. Levodopa in the treatment of Parkinson's disease: Current controversies. Mov Disord. 2004; 19(9):997.

2. Holloway RG, Shoulson I, Fahn S, et al. Pramipexole vs levodopa as initial treatment for Parkinson disease: A 4-year randomized controlled trial. Arch Neurol. 2004;61(7):1044-1053.

3. Rascol O, Brooks DJ, Korczyn AD, et al. A five-year study of the incidence of dyskinesia in patients with early Parkinson's disease who were treated with ropinirole or levodopa. 056 Study Group. $N$ Engl $J$ Med. 2000;342(20):1484-1491.

4. Rinne UK, Bracco F, Chouza C, et al. Early treatment of Parkinson's disease with cabergoline delays the onset of motor complications. Results of a double-blind levodopa controlled trial. The PKDS009 Study Group. Drugs. 1998;55 (Suppl 1):23-30.

5. Oertel WH, Wolters E, Sampaio C, et al. Pergolide versus levodopa monotherapy in early Parkinson's disease patients: The PELMOPET study. Mov Disord. 2005;21(3):343-353.

6. Clarke CE, Davies P. Systematic review of acute levodopa and apomorphine challenge tests in the diagnosis of idiopathic Parkinson's disease. J Neurol Neurosurg Psychiatry. 2000;69(5):590-594.

7. Goetz CG, Poewe W, Rascol O, et al. Movement Disorder Society Task Force report on the Hoehn and Yahr staging scale: Status and recommendations. Mov Disord. 2004;19(9):1020-1028.

8. Rajput AH. Levodopa prolongs life expectancy and is non-toxic to substantia nigra. Parkinsonism Relat Disord. 2001;8(2):95-100.

9. Hoehn MM. The natural history of Parkinson's disease in the prelevodopa and post-levodopa eras. Neurol Clin. 1992;10(2):331-339.

10. Nutt JG, Wooten GF. Clinical practice. Diagnosis and initial management of Parkinson's disease. N Engl J Med. 2005;353(10):1021-1027.

11. Parkinson Study Group. Pramipexole vs levodopa as initial treatment for Parkinson disease: A randomised controlled trial. JAMA. 2000; 284(15):1931-1938.

12. Kurlan R. "Levodopa phobia": A new iatrogenic cause of disability in Parkinson disease. Neurology. 2005;64(5):923-924.

13. Jenner P. Pathophysiology and biochemistry of dyskinesia: Clues for the development of non-dopaminergic treatments. J Neurol. 2000;247 (Suppl 2):II43-II50

14. Whone AL, Watts RL, Stoessl AJ, et al. Slower progression of Parkinson's disease with ropinirole versus levodopa: The REAL-PET study. Ann Neurol. 2003;54(1):93-101.

15. Rascol O, Brooks DJ, Korczyn AD, et al. Development of dyskinesias in a 5-year trial of ropinirole and L-dopa. Mov Disord. 2006;21(11): 1844-1850.

16. Parkinson Study Group. Long-term effect of initiating pramipexole vs levodopa in early Parkinson disease. Arch Neurol. 2009;66(5): 563-570.

17. Lees AJ, Katzenschlager R, Head J, Ben-Shlomo Y. Ten-year follow-up of three different initial treatments in de-novo PD: A randomized trial. Neurology. 2001;57(9):1687-1694. 
18. Hauser RA, Rascol O, Korczyn AD, et al. Ten-year follow-up of Parkinson's disease patients randomized to initial therapy with ropinirole or levodopa. Mov Disord. 2007;22(16):2409-2417.

19. Rascol O, Korczyn A, De Deyn PP, Lang A. Incidence of dyskinesias in a 10-year naturalistic follow-up of patients with early Parkinson's disease (PD) initially receiving ropinirole compared with L-dopa. Mov Disord. 2005;20 (Suppl 10):S63.

20. Hauser RA, Friedlander J, Zesiewicz TA, et al. A home diary to assess functional status in patients with Parkinson's disease with motor fluctuations and dyskinesia. Clin Neuropharmacol. 2000;23(2): 75-81.

21. Katzenschlager R, Head J, Schrag A, et al. Fourteen-year final report of the randomized PDRG-UK trial comparing three initial treatments in PD. Neurology. 2008;71(7):474-480.

22. Stocchi F, Rascol O, Kieburtz K, et al. Initiating levodopa/carbidopa therapy with and without entacapone in early Parkinson disease: the STRIDE-PD study. Ann Neurol. 2010;68(1):18-27.

23. Hung S, Adeli G, Arenovich T, Fox S, Lang A. Patient perception of dyskinesias in Parkinson's disease. Neurology. 2007;68(6):A232.

24. Larsen JP, Worm-Petersen J, Siden A, et al. The tolerability and efficacy of entacapone over 3 years in patients with Parkinson's disease. Eur J Neurol. 2003;10(2):137-146.

25. Olanow CW, Stern MB, Sethi K. The scientific and clinical basis for the treatment of Parkinson disease (2009). Neurology. 2009;72(21 Suppl4): S1-S136.

26. Obeso JA, Olanow CW, Nutt JG. Levodopa motor complications in Parkinson's disease. Trends Neurosci. 2000;23 (Suppl 10):S2-S7.

27. Antonini A, Tolosa E, Mizuno Y, Yamamoto M, Poewe WH. A reassessment of risks and benefits of dopamine agonists in Parkinson's disease. Lancet Neurol. 2009;8(10):929-937.

28. Weintraub D. Dopamine and impulse control disorders in Parkinson's disease. Ann Neurol. 2008;64 (Suppl 2):S93-S100.

29. Voon V, Hassan K, Zurowski M, et al. Prospective prevalence of pathologic gambling and medication association in Parkinson disease. Neurology. 2006;66(11):1750-1752.

30. Michel PP, Hefti F. Toxicity of 6-hydroxydopamine and dopamine for dopaminergic neurons in culture. J Neurosci Res. 1990;26(4): $428-435$.

31. Spencer JP, Jenner A, Aruoma OI, et al. Intense oxidative DNA damage promoted by L-dopa and its metabolites. Implications for neurodegenerative disease. FEBS Lett. 1994;353(3):246-250.

32. Mena MA, Pardo B, Casarejos MJ, Fahn S, Garcia de Yebenes J. Neurotoxicity of levodopa on catecholamine-rich neurons. Mov Disord. 1992;7(1):23-31.

33. Fahn S. Is levodopa toxic? Neurology. 1996;47 (Suppl 3):S184-S195.

34. Mena MA, Casarejos MJ, Carazo A, Paino CL, Garcia de Yebenes J. Glia conditioned medium protects fetal rat midbrain neurones in culture from L-DOPA toxicity. Neuroreport. 1996;7(2):441-445.

35. Mena MA, Casarejos MJ, Carazo A, Paino CL, Garcia de Yebenes J. Glia protect fetal midbrain dopamine neurons in culture from L-DOPA toxicity through multiple mechanisms. J Neural Transm. 1997; 104(4-5):317-328.

36. Pardo B, Mena MA, Fahn S, Garcia de Yebenes J. Ascorbic acid protects against levodopa-induced neurotoxicity on a catecholamine-rich human neuroblastoma cell line. Mov Disord. 1993;8(3):278-284.

37. Mena MA, Pardo B, Paino CL, De Yebenes JG. Levodopa toxicity in foetal rat midbrain neurones in culture: Modulation by ascorbic acid. Neuroreport. 1993;4(4):438-440.

38. Agid Y. Levodopa: Is toxicity a myth? Neurology. 1998;50(4):858-863.

39. Perry TL, Yong VW, Ito M, et al. Nigrostriatal dopaminergic neurons remain undamaged in rats given high doses of L-DOPA and carbidopa chronically. J Neurochem. 1984;43(4):990-993.

40. Pearce RK, Heikkila M, Linden IB, Jenner P. L-dopa induces dyskinesia in normal monkeys: Behavioural and pharmacokinetic observations Psychopharmacology (Berl). 2001;156(4):402-409.

41. Rajput AH, Fenton M, Birdi S, Macaulay R. Is levodopa toxic to human substantia nigra? Mov Disord. 1997;12(5):634-638.
42. Murer MG, Dziewczapolski G, Menalled LB, et al. Chronic levodopa is not toxic for remaining dopamine neurons, but instead promotes their recovery, in rats with moderate nigrostriatal lesions. Ann Neurol. 1998 ; 43(5):561-575.

43. Datla KP, Blunt SB, Dexter DT. Chronic L-DOPA administration is not toxic to the remaining dopaminergic nigrostriatal neurons, but instead may promote their functional recovery, in rats with partial 6-OHDA or $\mathrm{FeCl}(3)$ nigrostriatal lesions. Mov Disord. 2001;16(3):424-34.

44. Hagenah J, Klein C, Sieberer M, Vieregge P. Exogenous levodopa is not toxic to elderly subjects with non-parkinsonian movement disorders: Further clinical evidence. J Neural Transm. 1999;106(3-4):301-307.

45. Fahn S, Oakes D, Shoulson I, et al. Levodopa and the progression of Parkinson's disease. N Engl J Med. 2004;351(24):2498-2508.

46. Miyasaki JM, Martin W, Suchowersky O, Weiner WJ, Lang AE. Practice parameter: Initiation of treatment for Parkinson's disease: An evidencebased review: Report of the Quality Standards Subcommittee of the American Academy of Neurology. Neurology. 2002;58(1):11-17.

47. Poewe W, Wenning G. Levodopa in Parkinson's disease: Mechanisms of action and pathophysiology of late failure. In: Jankovic J, Tolosa E, editors. Parkinson's Disease and Movement Disorders. 4th ed. Philadelphia, PA: Lippincott Williams and Wilkins; 2002.

48. Stocchi F, Vacca L, Ruggieri S, Olanow CW. Intermittent vs continuous levodopa administration in patients with advanced Parkinson disease: A clinical and pharmacokinetic study. Arch Neurol. 2005;62(6):905-910.

49. Antonini A, Isaias IU, Canesi M, et al. Duodenal levodopa infusion for advanced Parkinson's disease: 12-month treatment outcome. Mov Disord. 2007;22(8):1145-1149.

50. Obeso JA, Rodriguez-Oroz MC, Chana P, et al. The evolution and origin of motor complications in Parkinson's disease. Neurology. 2000;55 (11 Suppl 4):S13-S20.

51. Obeso JA, Rodriguez-Oroz M, Marin C, et al. The origin of motor fluctuations in Parkinson's disease: Importance of dopaminergic innervation and basal ganglia circuits. Neurology. 2004;62(1 Suppl 1):S17-S30.

52. Olanow W, Schapira AH, Rascol O. Continuous dopamine-receptor stimulation in early Parkinson's disease. Trend Neurosci. 2000;23 (Suppl 10): S117-S126.

53. Stocchi F. The levodopa wearing-off phenomenon in Parkinson's disease: Pharmacokinetic considerations. Expert Opin Pharmacother. 2006;7(10):1399-1407.

54. Maratos EC, Jackson MJ, Pearce RK, Cannizzaro C, Jenner P. Both short- and long-acting D-1/D-2 dopamine agonists induce less dyskinesia than L-DOPA in the MPTP-lesioned common marmoset (Callithrix jacchus). Exp Neurol. 2003;179(1):90-102.

55. Gagnon C, Bedard PJ, Di Paolo T. Effect of chronic treatment of MPTP monkeys with dopamine D-1 and/or D-2 receptor agonists. Eur J Pharmacol. 1990;178(1):115-120.

56. Gomez-Mancilla B, Bedard PJ. Effect of chronic treatment with (+)-PHNO, a D2 agonist in MPTP-treated monkeys. Exp Neurol. 1992; 117(2):185-188.

57. Luquin MR, Laguna J, Herrero MT, Obeso JA. Behavioral tolerance to repeated apomorphine administration in parkinsonian monkeys J Neurol Sci. 1993;114(1):40-44.

58. Bedard PJ, Di Paolo T, Falardeau P, Boucher R. Chronic treatment with L-DOPA, but not bromocriptine induces dyskinesia in MPTPparkinsonian monkeys. Correlation with [3H]spiperone binding. Brain Res. 1986;379(2):294-299.

59. Maratos EC, Jackson MJ, Pearce RK, Jenner P. Antiparkinsonian activity and dyskinesia risk of ropinirole and L-DOPA combination therapy in drug naive MPTP-lesioned common marmosets (Callithrix jacchus) Mov Disord. 2001;16(4):631-641.

60. Hadj Tahar A, Gregoire L, Bangassoro E, Bedard PJ. Sustained cabergoline treatment reverses levodopa-induced dyskinesias in parkinsonian monkeys. Clin Neuropharmacol. 2000;23(4):195-202.

61. Blanchet PJ, Calon F, Martel JC, et al. Continuous administration decreases and pulsatile administration increases behavioral sensitivity to a novel dopamine D2 agonist (U-91356A) in MPTP-exposed monkeys. J Pharmacol Exp Ther. 1995;272(2):854-859. 
62. Morissette M, Goulet M, Soghomonian JJ, et al. Preproenkephalin mRNA expression in the caudate-putamen of MPTP monkeys after chronic treatment with the D2 agonist U91356A in continuous or intermittent mode of administration: Comparison with L-DOPA therapy. Brain Res Mol Brain Res. 1997;49(1-2):55-62.

63. Katzenschlager R, Hughes A, Evans A, et al. Continuous subcutaneous apomorphine therapy improves dyskinesias in Parkinson's disease: A prospective study using single-dose challenges. Mov Disord. 2005; 20(2):151-157.

64. Colzi A, Turner K, Lees AJ. Continuous subcutaneous waking day apomorphine in the long term treatment of levodopa induced interdose dyskinesias in Parkinson's disease. J Neurol Neurosurg Psychiatry. 1998;64(5):573-576.

65. Stocchi F, Ruggieri S, Vacca L, Olanow CW. Prospective randomized trial of lisuride infusion versus oral levodopa in patients with Parkinson's disease. Brain. 2002;125(Pt 9):2058-2066.

66. Syed N, Murphy J, Zimmerman T Jr, Mark MH, Sage JI. Ten years' experience with enteral levodopa infusions for motor fluctuations in Parkinson's disease. Mov Disord. 1998;13(2):336-338.

67. Nyholm D, Askmark H, Gomes-Trolin C, et al. Optimizing levodopa pharmacokinetics: Intestinal infusion versus oral sustained-release tablets. Clin Neuropharmacol. 2003;26(3):156-163.

68. Devos D. Patient profile, indications, efficacy and safety of duodenal levodopa infusion in advanced Parkinson's disease. Mov Disord. 2009; 24(7):993-1000

69. Annic A, Devos D, Seguy D, et al. [Continuous dopaminergic stimulation by Duodopa in advanced Parkinson's disease: Efficacy and safety]. Rev Neurol (Paris). 2009;165(8-9):718-27. French.

70. Brime B, Ballesteros MP, Frutos P. Preparation and in vitro characterization of gelatin microspheres containing Levodopa for nasal administration. J Microencapsul. 2000;17(6):777-784.

71. Babita K, Tiwary AK. Transcutaneous delivery of levodopa: Enhancement by fatty acid synthesis inhibition. Mol Pharm. 2005;2(1):57-63.
72. Dupont E, Andersen A, Boas J, et al. Sustained-release Madopar HBS compared with standard Madopar in the long-term treatment of de novo parkinsonian patients. Acta Neurol Scand. 1996;93(1):14-20.

73. Block G, Liss C, Reines S, Irr J, Nibbelink D. Comparison of immediate-release and controlled release carbidopa/levodopa in Parkinson's disease. A multicenter 5-year study. The CR First Study Group. Eur Neurol. 1997;37(1):23-27.

74. Koller WC, Hutton JT, Tolosa E, Capilldeo R. Immediate-release and controlled-release carbidopa/levodopa in PD: A 5-year randomized multicenter study. Carbidopa/Levodopa Study Group. Neurology. 1999; 53(5):1012-1019.

75. Brooks DJ. Optimizing levodopa therapy for Parkinson's disease with levodopa/carbidopa/entacapone: Implications from a clinical and patient perspective. Neuropsychiatr Dis Treat. 2008;4(1):39-47.

76. Rascol O, Goetz C, Koller W, Poewe W, Sampaio C. Treatment interventions for Parkinson's disease: An evidence based assessment. Lancet. 2002;359(9317):1589-1598.

77. Rinne UK, Larsen JP, Siden A, Worm-Petersen J. Entacapone enhances the response to levodopa in parkinsonian patients with motor fluctuations. NOMECOMT Study Group. Neurology. 1998;51(5):1309-1314.

78. Brooks DJ, Sagar H. Entacapone is beneficial in both fluctuating and non-fluctuating patients with Parkinson's disease: A randomised, placebo controlled, double blind, six month study. J Neurol Neurosurg Psychiatry. 2003;74(8):1071-1079.

79. Poewe WH, Deuschl G, Gordin A, Kultalahti ER, Leinonen M. Efficacy and safety of entacapone in Parkinson's disease patients with suboptimal levodopa response: A 6-month randomized placebo-controlled doubleblind study in Germany and Austria (Celomen study). Acta Neurologica Scand. 2002;105(4):245-255.

80. Parkinson Study Group. Entacapone improves motor fluctuations in levodopa-treated Parkinson's disease patients. Ann Neurol. 1997;42(5): $747-755$.
Clinical Interventions in Aging

\section{Publish your work in this journal}

Clinical Interventions in Aging is an international, peer-reviewed journal focusing on evidence-based reports on the value or lack thereof of treatments intended to prevent or delay the onset of maladaptive correlates of aging in human beings. This journal is indexed on PubMed Central, MedLine, the American Chemical Society's 'Chemical

\section{Dovepress}

Abstracts Service' (CAS), Scopus and the Elsevier Bibliographic databases. The manuscript management system is completely online and includes a very quick and fair peer-review system, which is all easy to use. Visit http://www.dovepress.com/testimonials.php to read real quotes from published authors. 